\title{
A Comprehensive Merging Scheme in Collaborative Spectrum Detection
}

\author{
Wei Qiu ${ }^{1, a}$, Changhua Yao ${ }^{2, b}$ \\ ${ }^{1}$ Chinese Satellite Maritime Tracking and Controlling Department, Jiangyin, China \\ ${ }^{2}$ Institute of Communications Engineering, PLA University of Science and Technology, Nanjing \\ 210007, China \\ ammnown003@163.com, bmmnown004@163.com
}

Keywords: comprehensive merging, collaborative spectrum detection, reporting cost.

\begin{abstract}
In this paper, we propose a novel comprehensive merging scheme in collaborative spectrum detection (CSD) to achieve better throughput performance, considering the diversity of the reporting channels. Unlicenced users (UUs) with good reporting channel quality transmit quantized self observation statistics to detection center (DC), and others report their self judgements. DC makes the final judgement by carrying out comprehensive merging. The closed-form expressions of throughput and detection performance is obtained in the proposed comprehensive merging scheme. The simulation shows that the comprehensive merging scheme can achieve better throughput performance than hard merging scheme or soft merging scheme.
\end{abstract}

\section{Introduction}

In the centralized collaborative energy sensing, the hard merging scheme collects 1-bit self judgement, i.e., presence or absence of PU, while the soft merging scheme collects multibit information of the detected energy, which provides a better performance than the hard merging scheme at the cost of increased reporting cost. The reporting cost can not be ignored when the self sensing data is reported to the DC through a common control channel, which is stringent bandwidth-limited for the efficient usage of limited spectrum resource[1],[2],[3].

There are some researches which study cost-throughput tradeoff in a CRN that consists of a number of the UUs employing energy detectors and a single judgement DC. Many methods have been proposed to reduce the reporting cost. Some introduce multibit quantization combining scheme[4],[5], to reduce the amount of data transmitted, some focus on the selection of the collaborative users[6], and [7] proposes a reporting order control scheme to reduce the reporting time. To our best knowledge, related researches all adopt either hard merging scheme or soft merging scheme. In fact, the attenuations of reporting channels are hardly same [1], to meet the bit error rate (BER) required by the reliable communication, the data rates would be different. In hard merging, UUs with high reporting data rate waste their reporting capability, and in soft merging scheme, UUs with low reporting data rate introduce large cost.

We propose a novel comprehensive merging scheme to achieve better throughput performance, considering the effect of different data rates of reporting channels between each UU and DC. UU with good reporting channel quality would transmit multibit quantized self observation statistics to DC (just like soft merging), and UU with bad reporting channel quality would transmit self judgement (1 bit information) to DC (just like hard merging). DC makes the final judgement by carrying out Comprehensive merging. In the proposed scheme, UU can balance the cost and its contribution to detection by choosing the better reporting method, according to its reporting channel quality. The simulation shows that the comprehensive merging scheme can outperform hard merging or soft merging scheme. 


\section{SYSTEM MODEL}

We consider the scenario of Fig.1. $U U_{i}(\mathrm{i}=1, \ldots, \mathrm{M}$, where $\mathrm{M}$ denotes the number of UUs) is the independent energy detector, and reports its self judgement or its quantized self observation statistics to DC via common control channel sequentially [1],[2]. Denote vector $R=\left[R_{1}, R_{2}, \ldots, R_{M}\right]$ as a high to low arrangement of reporting data rates. In the Comprehensive merging scenario proposed, $U U_{i}(\mathrm{i}=\mathrm{K}+1, \mathrm{~K}+2, \ldots, \mathrm{M})$ with bad reporting channel reports self judgement to $\mathrm{DC}, U U_{i}(\mathrm{i}=1$, $2, \ldots, K)$ with good reporting channel reports self observation statistics to DC. DC makes the final judgement by carrying out comprehensive merging.

All the UUs perform self Spectrum detection simultaneously in the sensing phase, and report self data to the DC sequentially in the reporting phase. Data of UUs are transmitted in the data transmission phase if the DC declares that the PU is inactive. The durations of the sensing phase is $\tau_{s}$, the durations of the reporting phase is $\tau_{r}$, and the reporting delay of each $U U_{i}$ is $\tau_{r i} \cdot \tau_{r i_{-} s}$ is the reporting delay of $U U_{i}$ if $U U_{i}$ reports self observation statistics, and $\tau_{r i_{-} h}$ is the reporting delay of $U U_{i}$ if $S U_{i}$ reports self judgement.

\section{PERFORMANCE ANALYSIS}

The DC receives two kinds of data reported by UUs according to system model proposed in section II, $U U_{i}(\mathrm{i}=1,2, \ldots, \mathrm{K})$ reports its self observation statistics to $\mathrm{DC}$, and $U U_{i}(\mathrm{i}=\mathrm{K}+1, \mathrm{~K}+2, \ldots, \mathrm{M})$ reports its self judgement to DC. We define $\bar{P}_{d}$ as the target probability of system detection with which the PUs are regarded as being sufficiently protected.

In the comprehensive merging scheme, for $U U_{i}(\mathrm{i}=1,2, \ldots, \mathrm{K})$, following the analysis of soft collaborative energy detection in [8], we use the test statistic given by

$$
T_{S-K}=\frac{1}{K N} \sum_{i=1}^{K} \sum_{n=1}^{N}\left|y_{i}(n)\right|^{2}
$$

According to central limit theorem, $T_{S-K}$ can be approximated as:

$$
\begin{gathered}
H_{0}: T_{S-K} \sim N\left(\sigma_{u}^{2}, \frac{1}{K N} \sigma_{u}^{4}\right), \\
H_{1}: T_{S-K} \sim N\left((\gamma+1) \sigma_{u}^{2}, \frac{1}{K N}(\gamma+1)^{2} \sigma_{u}^{4}\right),
\end{gathered}
$$

The probability of detection with $\mathrm{K}$ nodes soft merging $P_{d_{-} K}$ is given by

$$
P_{d_{-} K}=\operatorname{Pr}\left[Y(i)>\lambda_{K} \mid H_{1}\right]=Q\left(\frac{\lambda_{K}-(\gamma+1) \sigma_{u}^{2}}{(\gamma+1) \sigma_{u}^{2}} \sqrt{K N}\right),
$$

where $\lambda_{K}$ is the soft mergingdetection threshold.

To achieve the given target probability of system detection $\bar{P}_{d}$, according to the OR-rule, the relationship between $\bar{P}_{d}$ and $P_{d_{-} K}$ is

$$
\begin{aligned}
& \bar{P}_{d}=1-\left(1-P_{d_{-} l}\right)^{M-K}\left(1-P_{d_{-} K}\right), \\
& P_{d_{-} K}=1-\frac{\left(1-\bar{P}_{d}\right)}{\left(1-P_{d_{-} l}\right)^{M-K}},
\end{aligned}
$$

The $\mathrm{K}$ nodes soft mergingdetection threshold $\lambda_{K}$ is given by

$$
\lambda_{K}=(\gamma+1) \sigma_{u}^{2}\left(Q^{-1}\left(P_{d_{-} K}\right) / \sqrt{K N}+1\right),
$$

The probability of false warning of $\mathrm{K}$ soft mergingis given by 


$$
P_{f_{-} K}=\operatorname{Pr}\left[Y(i)>\lambda_{K} \mid H_{0}\right]=Q\left(\frac{\lambda_{K}-\sigma_{u}^{2}}{\sigma_{u}^{2}} \sqrt{K N}\right),
$$

The probability of system false warning $P_{f}^{K_{-}{ }^{M}}$ when K UUs report self observation statistics is given by

$$
\begin{aligned}
& P_{f}^{K_{-} M}=1-\left(1-P_{f_{-} l}\right)^{M-K}\left(1-P_{f_{-} K}\right) \\
& =1-\left(1-P_{f_{-} l}\right)^{M-K}\left(1-Q\left(\frac{\lambda_{K}-\sigma_{u}^{2}}{\sigma_{u}^{2}} \sqrt{K N}\right)\right. \\
& =1-\left(1-P_{f_{-} l}\right)^{M-K}\left(1-Q\left(\frac{(\gamma+1) \sigma_{u}^{2}\left(Q^{-1}\left(1-\frac{\left(1-\bar{P}_{d}\right)}{\left(1-P_{d_{-} l}\right)^{M-K}}\right) / \sqrt{K N}+1\right)-\sigma_{u}^{2}}{\sigma_{u}^{2}} \sqrt{K N}\right)\right.
\end{aligned}
$$

where $P_{f_{-} l}$ and $P_{d_{-} l}$ are related to $\bar{P}_{d}$. Fig.3 shows that $P_{f}^{K{ }_{-}{ }^{M}}$ decreases when $\mathrm{K}$ increases. In other words, in the comprehensive merging scheme, when the number of the UUs reporting their self observation statistics to DC increases, the accuracy of sensing result will be enhanced. When $U U_{i}$ $(\mathrm{i}=1,2, \ldots, \mathrm{K})$ reports its self observation statistics to $\mathrm{DC}$, and $S U_{i}(\mathrm{i}=\mathrm{K}+1, \mathrm{~K}+2, \ldots, \mathrm{M})$ reports its self judgement to DC, the total reporting delay is

$$
\tau_{r}=\sum_{i=1}^{K} \tau_{r i-s}+\sum_{i=K+1}^{M} \tau_{r i-h}=\sum_{i=1}^{K} \frac{D}{R_{i}}+\sum_{i=K+1}^{M} \frac{1}{R_{i}},
$$

The throughput is given by

$$
C(M, K)=\frac{T-\tau_{s}-\sum_{i=1}^{K} \frac{D}{R_{i}}+\sum_{i=K+1}^{M} \frac{1}{R_{i}}}{T}\left(1-P_{f}^{K{ }^{M}}\right) C_{0} \operatorname{Pr}\left(H_{0}\right),
$$

The relationship between the throughput $\mathrm{C}$ and $\mathrm{K}$ is obtained:

$$
\begin{aligned}
& C(M, K)=\frac{T-\tau_{s}-\sum_{i=1}^{K} \frac{D}{R_{i}}+\sum_{i=K+1}^{M} \frac{1}{R_{i}}}{T}\left(1-P_{f_{-} l}\right)^{M-K} \times \\
& \left(1-Q\left(\frac{(\gamma+1) \sigma_{u}^{2}\left(Q^{-1}\left(1-\frac{\left(1-\bar{P}_{d}\right)}{\left(1-P_{d_{-} l}\right)^{M-K}}\right) / \sqrt{K N}+1\right)-\sigma_{u}^{2}}{\sigma_{u}^{2}} \sqrt{K N}\right)\right) C_{0} \operatorname{Pr}\left(H_{0}\right)
\end{aligned}
$$

\section{Numerical Results and Discussions}

The system is set up as follows: The number of UUs is set to be $M=18$, and the fixed frame duration $\mathrm{T}=20 \mathrm{~ms}$. The sampling frequency is $6 \mathrm{MHz}$ and the $\tau_{s}$ is $1 \mathrm{~ms}$. The given target probability of system detection $\bar{P}_{d}$ is 0.99 . The SNR is $-18 \mathrm{~dB}$. We set quantization bits of observation statistics $\mathrm{D}=8$. 


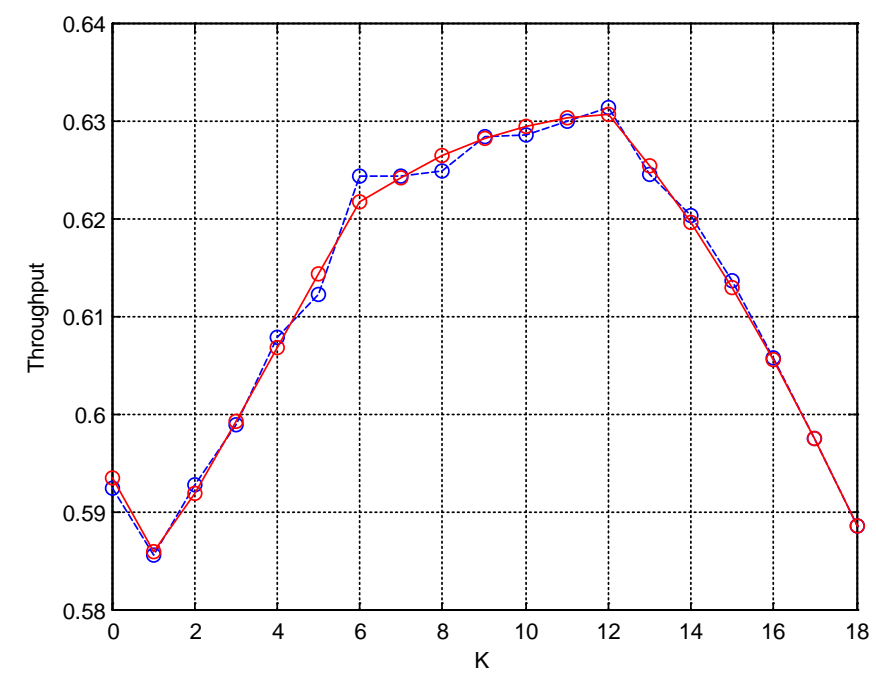

Fig.2 The simulation result.

Fig.2 shows that the proposed Comprehensive mergingscheme outperforms the hard mergingscheme or the soft mergingscheme, especially when the diversity of channel quality is obvious. The throughput first increases because the probability of system false warning decreases as K grows, and then the throughput decreases as K grows, because the cost increases fast when the UUs with bad reporting channel report their observation statistics rather than self judgements to DC. There is also throughput improvement when the data rates are the same.

\section{Summary}

We study cost-throughput tradeoff over different reporting channel quality in a collaborative network that consists of a number of the UUs employing energy detectors and a single judgement fusion center. We propose a novel comprehensive merging scheme which outperforms both hard mergingand soft merging scheme, especially when the diversity of the reporting channel is obvious. Moreover, even in the situation of same reporting channel quality, the throughput performance can benefit from the proposed scheme.

\section{References}

[1]. W Xia, W Yuan, W Cheng, W Liu, S Wang, J Xu, “Optimization of cooperative

Spectrum detection in ad-hoc cognitive radio networks,” in Proceedings of the

IEEE Global Telecommunications Conference 2010, pp.1-5.

[2] Huogen $\mathrm{Yu}^{*}$, Wanbin Tang and Shaoqian Li, “Optimization of collaborativeSpectrum detection with sensing user selection in cognitive radio networks”, Journal on Wireless Communications and Networking, 2011.

[3] Li-Chun Wang ; Yin-Chih Lu ; Chung-Wei Wang ; Wei, D.S.L, "Latency Analysis for Dynamic Spectrum Access in Cognitive Radio: Dedicated or Embedded Control Channel? ”, IEEE 18th International Symposium on Personal, Indoor and Mobile Radio Communications, 2007, pp.1-5.

[4] Babak Abbasi Bastami and Ebrahim Saberinia, “A Practical Multibit Data Combining Strategy for CollaborativeSpectrum detection,” IEEE TRANSACTIONS ON VEHICULAR TECHNOLOGY, VOL. 62, NO. 1, JANUARY 2013

[5] Y. Tani and T. Saba, "Quantization scheme for energy detector of soft judgement collaborativeSpectrum detection in cognitive radio.” IEEE Global Telecommunications Conference 2010, pp.69-73. 
[6] Wha Sook Jeon, Dong Heon Lee, Dong Geun Jeong,"Collaborative Sensing Management for Cognitive Radio Networks with Reporting Cost," IEEE TRANSACTIONS ON WIRELESS COMMUNICATIONS, VOL. 12, NO. 2, FEBRUARY 2013

[7]. Gosan Noh, Hano Wang, Junho Jo, Byoung-Hoon Kim, Daesik Hong, "Reporting Order Control for Fast Primary Detection in CollaborativeSpectrum detection,” IEEE TRANSACTIONS ON VEHICULAR TECHNOLOGY, VOL. 60, NO. 8, OCTOBER 2011.

[8] S. M. Mishra, A. Sahai, and R. W. Broderson, "Collaborativesensing among cognitive radios," IEEE ICC 2006, VOL.4 pp.1658-1663. 